\title{
Brane world effective action at low energies and AdS/CFT correspondence
}

$\operatorname{AUTHOR}(\mathrm{S})$ :

Kanno, S; Soda, J

CITATION:

Kanno, S ...[et al]. Brane world effective action at low energies and AdS/CFT correspondence. PHYSICAL REVIEW D 2002, 66(4): 043526.

ISSUE DATE:

2002-08-15

URL:

http://hdl.handle.net/2433/49934

RIGHT:

Copyright 2002 American Physical Society 
PHYSICAL REVIEW D 66, 043526 (2002)

\title{
Brane world effective action at low energies and AdS/CFT correspondence
}

\author{
Sugumi Kanno* \\ Graduate School of Human and Environment Studies, Kyoto University, Kyoto 606-8501, Japan \\ Jiro Soda \\ Department of Fundamental Sciences, FIHS, Kyoto University, Kyoto 606-8501, Japan
}

(Received 24 May 2002; published 26 August 2002)

\begin{abstract}
A low energy iteration scheme to study nonlinear gravity in the brane world is developed. As a result, we obtain the brane world effective action at low energies. The relation between the geometrical approach and the approach using the AdS/CFT correspondence is also clarified. In particular, we find generalized dark radiation as homogeneous solutions in our iteration scheme. Moreover, the precise correspondence between the bulk geometry and the brane effective action is established, which gives a holographic view of the brane world.
\end{abstract}

DOI: 10.1103/PhysRevD.66.043526

\section{INTRODUCTION}

The brane world scenario has been the subject of intensive investigation for the past few years. In particular, Randall and Sundrum proposed a simple model where the fourdimensional brane with tension $\sigma$ is embedded in the fivedimensional asymptotically anti-de Sitter (AdS) bulk with a curvature scale $l$. The model is described by the action

$$
\begin{aligned}
S= & \frac{1}{2 \kappa^{2}} \int d^{5} x \sqrt{-g}\left(\mathcal{R}+\frac{12}{l^{2}}\right)-\sigma \int d^{4} x \sqrt{-h} \\
& +\int d^{4} x \sqrt{-h} \mathcal{L}_{\text {matter }},
\end{aligned}
$$

where $\mathcal{R}$ and $\kappa^{2}$ are the scalar curvature and gravitational constant in five dimensions, respectively. We impose $Z_{2}$ symmetry on this spacetime, with the brane at the fixed point ( $y=0$ in the coordinate system used later). Throughout this paper, $h_{\mu \nu}$ represents the induced metric on the brane. They showed that, in spite of the noncompact extra dimension, the gravity is localized on the brane at the linearized level [1-3]. Consequently, the conventional linearized Einstein equation approximately holds at scales large compared with the curvature scale $l$. The cosmology in the context of this model has also been investigated enthusiastically [4-19]. It turns out that there is a nonconventional quadratic term of the energy density at high energies and, even in the low energy regime, there exists dark radiation caused by the black hole in the bulk. This dark radiation component is also found in the cosmological perturbations [20,21].

It is desirable to extend this understanding of gravity in the brane world to general nonlinear cases. In order to understand nonlinear gravity in the brane world scenario, Shiromizu et al. proposed an elegant geometrical approach [22]. They obtained the "effective" four-dimensional equations

$$
G_{\mu \nu}^{(4)}=8 \pi G_{N} T_{\mu \nu}+\kappa^{4} \pi_{\mu \nu}-E_{\mu \nu}
$$

\footnotetext{
*Electronic address: kanno@phys.h.kyoto-u.ac.jp

†Electronic address: jiro@phys.h.kyoto-u.ac.jp
}

PACS number(s): 98.80.Cq, 04.50.+h, 98.80.Hw

where $T_{\mu \nu}$ is the energy-momentum tensor of matter, $E_{\mu \nu}$ is the projection of the Weyl tensor $\left.C_{y \mu y \nu}\right|_{y=0}, G_{N}$ denotes Newton's constant, and

$$
\begin{aligned}
\pi_{\mu \nu}= & -\frac{1}{4} T_{\mu}^{\lambda} T_{\lambda \nu}+\frac{1}{12} T T_{\mu \nu} \\
& +\frac{1}{8} g_{\mu \nu}\left(T^{\alpha \beta} T_{\alpha \beta}-\frac{1}{3} T^{2}\right) .
\end{aligned}
$$

Here the relations

$$
\kappa^{2} \sigma=\frac{6}{l}, \quad \frac{\kappa^{2}}{l}=8 \pi G_{N}
$$

have been assumed. From their effective equation of motion, one can see the "electric" part of the Weyl tensor $E^{\mu}{ }_{\nu}$ which characterizes the bulk geometry effects on the brane dynamics. Conversely, the matter on the brane changes the bulk geometry, as can be seen from the equation $\nabla_{\mu} E^{\mu}{ }_{\nu}$ $=\kappa^{4} \nabla_{\mu} \pi^{\mu}{ }_{\nu}$ derived through the Bianchi identity. However, it should be noted that it is by no means a closed system of equations. We need to solve the bulk geometry to determine $E_{\mu \nu}$ completely.

Unfortunately, it is a formidable task to solve the fivedimensional Einstein equation exactly. However, notice that typically the length scale of the internal space is $l$ $\ll 0.1 \mathrm{~mm}$. On the other hand, the usual astrophysical and cosmological phenomena take place at a scale larger than this scale. Then we need only a low energy effective theory to analyze a variety of problems, for example, the formation of the black hole, the propagation of the gravitational wave, the evolution of the cosmological perturbation, and so on. It should be stressed that low energy does not necessarily imply weak gravity.

It has been suggested that gravity on the brane at low energies can be understood through the AdS conformal field 
theory (CFT) correspondence [23]. From the correspondence, one can guess the effective equations of motion on the brane as

$$
G_{\mu \nu}^{(4)}=8 \pi G_{N}\left(T_{\mu \nu}+T_{\mu \nu}^{C F T}\right)+\left\{R^{2} \text { terms }\right\},
$$

where the $R^{2}$ terms represent the higher order curvature terms and $T^{C F T}$ denotes the energy-momentum tensor of the cutoff version of conformal field theory. However, no one knows what is the cutoff CFT. Moreover, it should be noted that the AdS/CFT correspondence is a specific conjecture. Indeed, originally, Maldacena conjectured that the supergravity on $\operatorname{AdS}_{5} \times S^{5}$ is dual to the four-dimensional $\mathcal{N}=4$ super Yang-Mills theory [24]. We should be careful not to use such a conjecture thoughtlessly. Nevertheless, the AdS/CFT correspondence seems to be related to the brane world model as has been demonstrated by several people [25-29].

Since both the geometrical and AdS/CFT approaches seem to have their own merit, it would be beneficial to understand the mutual relationship. Recently, Shiromizu and Ida tried to understand the AdS/CFT correspondence from the geometrical point of view [30]. They argued that $\pi^{\mu}{ }_{\mu}$ corresponds to the trace anomaly of the cutoff CFT on the brane. However, this result is rather paradoxical because there exists no trace anomaly in an odd dimensional brane although $\pi_{\mu}^{\mu}$ exists even in that case. Thus, the more precise relation between the geometrical and the AdS/CFT approaches remains to be understood.

In this paper, we derive the effective four-dimensional theory without using any concept of the AdS/CFT correspondence a priori. Thus, we avoid using the vague concept of cutoff CFT. To solve the five-dimensional equations of motion, we use a low energy iteration scheme. In particular, we impose the Dirichlet boundary condition at the brane position, in contrast to the AdS/CFT approach where the Dirichlet boundary condition is imposed at infinity. We also consider the "constant" of the integration, i.e., homogeneous solutions, carefully. As a consequence, we show that the dark radiation can be understood from the holographic point of view. Moreover, the relation between the geometrical and AdS/CFT approaches is uncovered. The correspondence between the bulk geometry and the brane effective action is also explicitly found.

This paper is organized as follows. In Sec. II, we develop the iteration scheme to solve the Einstein equations at low energies. In Sec. III, we derive the brane effective action from the junction condition. We see the effective equation does not reduce to the conventional Einstein equation even in the low energy regime. This is due to the generalized dark radiation found in this paper. It is also found that the nonlocal part of the effective equation is represented by the energy-momentum tensor with a trace part coinciding with the trace anomaly of CFT. Section IV is devoted to the conclusion. In the Appendix, we analyze the $(d+1)$-dimensional case because the result is qualitatively different from the five-dimensional case.

\section{LOW ENERGY ITERATION SCHEME}

In the Gaussian normal coordinate system, the geometry of the brane world is described by

$$
d s^{2}=d y^{2}+g_{\mu \nu}\left(y, x^{\mu}\right) d x^{\mu} d x^{\nu} .
$$

Note that the brane is located at $y=0$ in this coordinate system. Then, the five-dimensional Einstein equation in the bulk becomes

$$
\begin{aligned}
K_{\nu, y}^{\mu} & -K K_{\nu}^{\mu}+R_{\nu}^{\mu} \\
& =-\frac{4}{l^{2}} \delta_{\nu}^{\mu}+\kappa^{2}\left(\frac{1}{3} \sigma \delta_{\nu}^{\mu}+T^{\mu}{ }_{\nu}-\frac{1}{3} \delta_{\nu}^{\mu} T\right) \delta(y),
\end{aligned}
$$

$$
\begin{gathered}
K_{, y}-K^{\alpha \beta} K_{\alpha \beta}=-\frac{4}{l^{2}}-\frac{\kappa^{2}}{3}(-4 \sigma+T) \delta(y), \\
\nabla_{\nu} K_{\mu}{ }^{\nu}-\nabla_{\mu} K=0,
\end{gathered}
$$

where $R^{\mu}{ }_{\nu}{ }^{(4)}$ is the curvature on the brane and $\nabla_{\mu}$ denotes the covariant derivative with respect to the metric $g_{\mu \nu}$. One can read off the junction condition from the above equations as

$$
\left.\left[K_{\nu}^{\mu}-\delta_{\nu}^{\mu} K\right]\right|_{y=0}=\frac{\kappa^{2}}{2}\left(-\sigma \delta_{\nu}^{\mu}+T_{\nu}^{\mu}\right)
$$

Recall that we are considering the $Z_{2}$ symmetric spacetime. Decomposing the extrinsic curvature into the traceless part and the trace part

$$
K_{\mu \nu}=\Sigma_{\mu \nu}+\frac{1}{4} h_{\mu \nu} K, \quad K=-\frac{\partial}{\partial y} \log \sqrt{-g},
$$

we obtain the basic equations which hold in the bulk:

$$
\begin{aligned}
& \Sigma_{\nu, y}^{\mu}-K \Sigma_{\nu}^{\mu}=-\left[\begin{array}{l}
(4) \\
R^{\mu}{ }_{\nu}-\frac{1}{4} \delta^{\mu}{ }_{\nu} R
\end{array}\right], \\
& \frac{3}{4} K^{2}-\Sigma^{\alpha}{ }_{\beta} \Sigma^{\beta}{ }_{\alpha}=\left[\begin{array}{c}
(4) \\
R
\end{array}\right]+\frac{12}{l^{2}}, \\
& K_{, y}-\frac{1}{4} K^{2}-\Sigma^{\alpha \beta} \Sigma_{\alpha \beta}=-\frac{4}{l^{2}}, \\
& \nabla_{\lambda} \Sigma_{\mu}^{\lambda}-\frac{3}{4} \nabla_{\mu} K=0 .
\end{aligned}
$$

The problem now is separated into two parts. First, we will solve the bulk equations of motion with the Dirichlet boundary condition at the brane, $g_{\mu \nu}\left(y=0, x^{\mu}\right)=h_{\mu \nu}\left(x^{\mu}\right)$. After that, the junction condition will be imposed at the brane. As it is the condition for the induced metric $h_{\mu \nu}$, it is naturally interpreted as the effective equations of motion for gravity on the brane.

Along the normal coordinate $y$, the metric varies with a characteristic length scale $l ; g_{\mu \nu, y} \sim g_{\mu \nu} / l$. Denote the char- 
acteristic length scale of the curvature fluctuation on the brane as $L$; then we have $R \sim g_{\mu \nu} / L^{2}$. For the reader's reference, let us take $l=1 \mathrm{~mm}$, for example. Then, the relations (4) give the scale, $\kappa^{2}=\left(10^{8} \mathrm{GeV}\right)^{-3}$ and $\sigma=1 \mathrm{TeV}^{4}$. In this paper, we will consider the low energy regime in the sense that the energy density of matter, $\rho$, on the brane is smaller than the brane tension, i.e., $\rho / \sigma \ll 1$. In this regime, a simple dimensional analysis

$$
\frac{\rho}{\sigma} \sim \frac{l\left(\kappa^{2} / l\right) \rho}{\kappa^{2} \sigma} \sim\left(\frac{l}{L}\right)^{2} \ll 1
$$

implies that the curvature on the brane can be neglected compared with the extrinsic curvature at low energies. Here, we have used the relations (4) and Einstein's equation on the brane, $R \sim g_{\mu \nu} / L^{2} \sim G_{N} \rho$. Thus, the anti-Newtonian or gradient expansion method used in the cosmological context is applicable to our problem [31-34]. The iteration scheme consists in writing the metric $g_{\mu \nu}$ as a sum of local tensors built out of the induced metric on the brane, the number of gradients increasing with the order. Hence, we will seek the metric as a perturbative series

$$
\begin{aligned}
g_{\mu \nu}\left(y, x^{\mu}\right)= & a^{2}(y)\left[h_{\mu \nu}\left(x^{\mu}\right)+g_{\mu \nu}^{(1)}\left(y, x^{\mu}\right)\right. \\
& \left.+g_{\mu \nu}^{(2)}\left(y, x^{\mu}\right)+\cdots\right], \\
g_{\mu \nu}^{(i)}\left(y=0, x^{\mu}\right)= & 0,
\end{aligned}
$$

where $a^{2}(y)=\exp (-2 y / l)$ is extracted for reasons explained later and we put the Dirichlet boundary condition $g_{\mu \nu}(y$ $=0, x)=h_{\mu \nu}(x)$ at the brane. Other quantities are also expanded as

$$
K_{\nu}^{\mu}=K_{\nu}^{(0) \mu}+K_{\nu}^{(1) \mu}+K_{\nu}^{(2) \mu}+\cdots .
$$

Our scheme is different from the calculation usually performed in the AdS/CFT correspondence in that the Dirichlet boundary condition is imposed not at infinity but at the finite point $y=0$, the location of the brane. Furthermore, we carefully keep the constants of integration, i.e., homogeneous solutions. These homogeneous solutions are ignored in the calculation of AdS/CFT correspondence. However, they play an important role in the brane world.

\section{A. Zeroth order}

At zeroth order, we can neglect the curvature term. Then we have

$$
\begin{aligned}
\Sigma_{\nu, y}^{(0) \mu}-K_{\nu}^{(0)} \Sigma_{\nu}^{(0) \mu} & =0, \\
\frac{3}{4} K^{(0) 2}-\Sigma_{\beta}^{(0) \alpha} \Sigma_{\alpha}^{(0) \beta} & =\frac{12}{l^{2}}, \\
K_{, y}^{(0)}-\frac{1}{4} K^{(0) 2}-\Sigma^{(0) \alpha \beta} \Sigma_{\alpha \beta}^{(0)} & =-\frac{4}{l^{2}}, \\
\nabla_{\lambda} \Sigma_{\mu}^{(0) \lambda}-\frac{3}{4} \nabla_{\mu} K^{(0)} & =0 .
\end{aligned}
$$

Equation (20) can be readily integrated into

$$
\Sigma_{\nu}^{(0) \mu}=\frac{C_{\nu}^{\mu}\left(x^{\mu}\right)}{\sqrt{-g}}, \quad C_{\mu}^{\mu}=0,
$$

where $C^{\mu}{ }_{\nu}$ is the integration "constant." Equation (23) also requires $C^{\mu}{ }_{\nu \mid \mu}=0$. It represents a radiationlike fluid on the brane. Although this deserves further investigation, the calculation is complicated. Furthermore, this term is not relevant to our realistic universe because it represents a strongly anisotropic universe. Indeed, as we see later, this term must vanish in order to satisfy the junction condition. Therefore, we simply put $C^{\mu}{ }_{\nu}=0$, hereafter. Now, it is easy to solve the remaining equations. The result is

$$
K^{(0)}=\frac{4}{l} .
$$

Using the definition of the extrinsic curvature

$$
K_{\mu \nu}^{(0)}=-\frac{1}{2} \frac{\partial}{\partial y} g_{\mu \nu}^{(0)},
$$

we get the zeroth order metric as

$$
d s^{2}=d y^{2}+a^{2}(y) h_{\mu \nu}\left(x^{\mu}\right) d x^{\mu} d x^{\nu}, \quad a(y)=e^{-2 y / l},
$$

where the tensor $h_{\mu \nu}$ is the induced metric on the brane.

\section{B. First order}

The next order solutions are obtained by taking into account the terms neglected at zeroth order. At first order, Eqs. (12)-(15) become

$$
\begin{aligned}
\sum_{\nu, y}^{(1) \mu}-\frac{4}{l} \Sigma_{\nu}^{(1) \mu} & =-\left[\begin{array}{c}
(4) \\
R_{\nu} \\
\nu
\end{array}-\frac{1}{4} \delta_{\nu}^{\mu}{ }^{(4)}\right]^{(1)}, \\
\frac{6}{l} K^{(1)} & =\left[\begin{array}{c}
(4) \\
R
\end{array}\right]^{(1)}, \\
K_{, y}^{(1)}-\frac{2}{l} K^{(1)} & =0, \\
\Sigma_{\mu}^{(1) \lambda \lambda}-\frac{3}{4} K_{\mid \mu}^{(1)} & =0,
\end{aligned}
$$

where the superscript (1) represents the order of the derivative expansion and $\mid$ denotes the covariant derivative with respect to the metric $h_{\mu \nu}$. Here, $\left[R_{\nu}^{\mu}\right]^{(1)}$ means that the curvature is approximated by taking the Ricci tensor of $a^{2} h_{\mu \nu}$ in place of $R_{\nu}^{(4) \mu}$. It is also convenient to write it in terms of the Ricci tensor of $h_{\mu \nu}$, denoted $R_{\nu}^{\mu}(h)$.

Substituting the zeroth order metric into $R^{(4)}$, we obtain

$$
K^{(1)}=\frac{l}{6 a^{2}} R(h) .
$$


Hereafter, we omit the argument of the curvature for simplicity. Simple integration of Eq. (28) also gives the traceless part of the extrinsic curvature as

$$
\Sigma_{\nu}^{(1) \mu}=\frac{l}{2 a^{2}}\left(R_{\nu}^{\mu}-\frac{1}{4} \delta_{\nu}^{\mu} R\right)+\frac{\chi_{\nu}^{\mu}(x)}{a^{4}},
$$

where the homogeneous solution satisfies the constraints $\chi_{\mu}^{\mu}=0$ and $\chi_{\nu \mid \mu}^{\mu}=0$. As we see later, this term corresponds to dark radiation at this order. The metric can be obtained as

$$
g_{\mu \nu}^{(1)}=-\frac{l^{2}}{2}\left(\frac{1}{a^{2}}-1\right)\left(R_{\mu \nu}-\frac{1}{6} h_{\mu \nu} R\right)-\frac{l}{2}\left(\frac{1}{a^{4}}-1\right) \chi_{\mu \nu},
$$

where we have imposed the boundary condition $g_{\mu \nu}^{(1)}(y$ $\left.=0, x^{\mu}\right)=0$. This $\chi$ field is essential to understanding the origin of the dark radiation from the holographic point of view.

\section{Second order}

In this subsection, we do not include the $\chi$ field because it complicates the equations. This is a consistent truncation procedure. Of course, we have calculated the second order solutions with the contribution of the $\chi$ field. They include terms such as $\chi^{\mu}{ }_{\nu} \chi^{\nu}{ }_{\mu}$, etc.

At second order, the basic equations become

$$
\begin{aligned}
& \sum_{\nu, y}^{(2) \mu}-\frac{4}{l} \Sigma_{\nu}^{(2) \mu}=-\left[R_{\nu}^{(4)}{ }_{\nu}-\frac{1}{4} \delta_{\nu}^{\mu} R\right]^{(4)}+K^{(1)} \Sigma_{\nu}^{(1) \mu} \\
& K^{(2)}= \frac{l}{6}\left[-\frac{3}{4} K^{(1) 2}+\Sigma_{\beta}^{(1) \alpha} \Sigma_{\alpha}^{(1) \beta}\right. \\
&\left.+\left[\begin{array}{c}
(4) \\
R
\end{array}\right] \text { (2) }\right] \\
& K_{, y}^{(2)}-\frac{2}{l} K^{(2)}= \frac{1}{4} K^{(1) 2}+\Sigma^{(1) \alpha \beta} \Sigma_{\alpha \beta}^{(1)} \\
& \Sigma_{\mu}^{(2) \lambda} \lambda-\frac{3}{4} K_{\mid \mu}^{(2)}+\Gamma_{\lambda \alpha}^{(1) \alpha} \Sigma_{\mu}^{(1) \lambda}-\Gamma_{\alpha \mu}^{(1) \lambda} \Sigma_{\lambda}^{\alpha}=0
\end{aligned}
$$

Substituting the solution up to first order into the Ricci tensor and picking up the second order quantities, we obtain

$$
\begin{aligned}
{\left[{ }^{(4)}{ }_{\nu}^{\mu}-\frac{1}{4} \delta_{\nu}^{\mu} R\right]^{(4)}=} & \frac{l^{2}}{2}\left(\frac{1}{a^{4}}-\frac{1}{a^{2}}\right)\left[R_{\alpha}^{\mu} R_{\nu}^{\alpha}-\frac{1}{6} R R_{\nu}^{\mu}\right. \\
& -\frac{1}{4} \delta_{\nu}^{\mu}\left(R^{\alpha}{ }_{\beta} R^{\beta}{ }_{\alpha}-\frac{1}{6} R^{2}\right) \\
& -\frac{1}{2}\left(R^{\alpha \mu}{ }_{\nu \alpha}+R_{\nu}^{\alpha}{ }_{\nu}{ }_{\mid \alpha}\right)+\frac{1}{3} R_{\mid \nu}^{\mid \mu} \\
& \left.+\frac{1}{2} \square R^{\mu}{ }_{\nu}-\frac{1}{12} \delta_{\nu}^{\mu} \square R\right],
\end{aligned}
$$

$$
\left[\begin{array}{c}
(4) \\
R
\end{array}\right]^{(2)}=\frac{l^{2}}{2}\left(\frac{1}{a^{4}}-\frac{1}{a^{2}}\right)\left[R_{\beta}^{\alpha} R_{\alpha}^{\beta}-\frac{1}{6} R^{2}\right],
$$

where we have used the formula $\delta R_{\mu \nu}=1 / 2\left[\delta g^{\alpha}{ }_{\mu \mid \nu \alpha}\right.$ $\left.+\delta g^{\alpha}{ }_{\mu \mid \nu \alpha}-\delta g_{\mid \mu \nu}-\delta g_{\mu \nu \mid \alpha}{ }^{\mid \alpha}\right]$. Using the above formula, the trace part is deduced algebraically as

$$
K^{(2)}=\frac{l^{3}}{8 a^{4}}\left(R_{\beta}^{\alpha} R_{\alpha}^{\beta}-\frac{2}{9} R^{2}\right)-\frac{l^{3}}{12 a^{2}}\left(R_{\beta^{\alpha}}^{\alpha}{ }_{\alpha}-\frac{1}{6} R^{2}\right) .
$$

By integrating the equation for the traceless part, we have

$$
\begin{aligned}
\Sigma_{\nu}^{(2) \mu}= & -\frac{l^{2}}{2}\left(\frac{y}{a^{4}}+\frac{l}{2 a^{2}}\right) \mathcal{S}_{\nu}^{\mu}-\frac{l^{3}}{24 a^{2}}\left(R R_{\nu}^{\mu}-\frac{1}{4} \delta_{\nu}^{\mu} R^{2}\right) \\
& +\frac{l^{3}}{a^{4}} t_{\nu}^{\mu},
\end{aligned}
$$

where we have defined the quantity

$$
\begin{aligned}
\mathcal{S}_{\nu}^{\mu}= & R_{\alpha}^{\mu} R_{\nu}^{\alpha}-\frac{1}{3} R R_{\nu}^{\mu}-\frac{1}{4} \delta_{\nu}^{\mu}\left(R_{\beta}^{\alpha} R_{\alpha}^{\beta}-\frac{1}{3} R^{2}\right) \\
& -\frac{1}{2}\left(R^{\alpha \mu}{ }_{\mid \nu \alpha}+R_{\nu}^{\alpha}{ }_{\nu}{ }_{\mid \alpha}-\frac{2}{3} R^{\mid \mu}{ }_{\mid \nu}-\square R^{\mu}{ }_{\nu}+\frac{1}{6} \delta_{\nu}^{\mu} \square R\right),
\end{aligned}
$$

which is transverse and traceless,

$$
\mathcal{S}^{\mu}{ }_{\nu \mid \mu}=0, \quad \mathcal{S}_{\mu}^{\mu}=0 .
$$

It is also useful to notice that this tensor can be derived from

$$
\delta \int d^{4} x \sqrt{-h} \frac{1}{2}\left[R^{\alpha \beta} R_{\alpha \beta}-\frac{1}{3} R^{2}\right]=\int d^{4} x \sqrt{-h} \mathcal{S}_{\mu \nu} \delta g^{\mu \nu} .
$$

The homogeneous solution $t^{\mu}{ }_{\nu}$ must be determined so that Eq. (38) holds. To be more precise, we must solve the constraint equation

$$
t_{\nu \mid \mu}^{\mu}-\frac{1}{16} R_{\beta}^{\alpha} R_{\alpha \mid \nu}^{\beta}+\frac{1}{48} R R_{\mid \nu}-\frac{1}{24} R_{\mid \lambda} R_{\nu}^{\lambda}=0 .
$$

As one can see immediately, there are ambiguities in integrating this equation. Indeed, there are two types of covariant local tensor whose divergences vanish:

$$
\begin{aligned}
\mathcal{H}^{\mu}{ }_{\nu}= & R^{\mu}{ }_{\alpha} R_{\nu}^{\alpha}{ }_{\nu}-\frac{1}{4} \delta_{\nu}^{\mu} R_{\beta}^{\alpha} R_{\alpha}{ }_{\alpha} \\
& -\frac{1}{2}\left(R^{\alpha \mu}{ }_{\nu \alpha}+R_{\nu}^{\alpha}{ }_{\nu}^{\mid \mu}{ }_{\mid \alpha}-\square R^{\mu}{ }_{\nu}-\frac{1}{2} \delta_{\nu}^{\mu} \square R\right), \\
\mathcal{K}^{\mu}{ }_{\nu}= & R R^{\mu}{ }_{\nu}-\frac{1}{4} \delta_{\nu}^{\mu} R^{2}-R^{\mid \mu}{ }_{\mid \nu}+\delta_{\nu}^{\mu} \square R .
\end{aligned}
$$

These terms come from the variation of the action 


$$
\begin{array}{r}
\delta \int d^{4} x \sqrt{-h} \frac{1}{2} R^{\alpha \beta} R_{\alpha \beta}=\int d^{4} x \sqrt{-h} \mathcal{H}_{\mu \nu} \delta g^{\mu \nu}, \\
\delta \int d^{4} x \sqrt{-h} \frac{1}{2} R^{2}=\int d^{4} x \sqrt{-h} \mathcal{K}_{\mu \nu} \delta g^{\mu \nu},
\end{array}
$$

respectively. Notice that $\mathcal{S}^{\mu}{ }_{\nu}=\mathcal{H}^{\mu}{ }_{\nu}-\mathcal{K}^{\mu}{ }_{\nu} / 3$. Thanks to the Gauss-Bonnet topological invariant, we do not need to consider the Riemann squared term. In addition to these local tensors, we have to take into account the nonlocal tensor $\tau^{\mu}{ }_{\nu}$ with the property $\tau_{\nu \mid \mu}^{\mu}=0$. Thus, we get

$$
\begin{aligned}
t_{\nu}^{\mu}= & \frac{1}{32} \delta_{\nu}^{\mu}\left(R_{\beta}^{\alpha} R_{\alpha}^{\beta}-\frac{1}{3} R^{2}\right) \\
& +\frac{1}{24}\left(R R_{\nu}^{\mu}-\frac{1}{4} \delta_{\nu}^{\mu} R^{2}\right)+\tau_{\nu}^{\mu}+\alpha \mathcal{S}_{\nu}^{\mu}+\frac{\beta}{3} \mathcal{K}^{\mu}{ }_{\nu},
\end{aligned}
$$

where the constants $\alpha$ and $\beta$ represents the freedom of the gravitational wave in the bulk. The condition $t_{\mu}^{\mu}=0$ leads to

$$
\tau_{\mu}^{\mu}=-\frac{1}{8}\left(R_{\beta}^{\alpha} R_{\alpha}^{\beta}-\frac{1}{3} R^{2}\right)-\beta \square R .
$$

The quantity $\tau^{\mu}{ }_{\nu}$ cannot be written in the local covariant form. Hence, this part is interpreted as the CFT in the context of the AdS/CFT correspondence.

\section{D. $n$th order}

In principle, we can continue our analysis up to a desired order using the following recursive formulas:

$$
\begin{aligned}
& \Sigma_{\nu}^{(n) \mu}=-\frac{1}{a^{4}} \int d y a^{4}\left\{\left[\begin{array}{l}
(4) \\
R^{\mu}{ }_{\nu}-\frac{1}{4} \delta_{\nu}^{\mu} R
\end{array}\right]^{(4)}\right. \\
& \left.-\sum_{p=1}^{n-1} K^{(p)} \Sigma_{\nu}^{(n-p) \mu}\right\} \\
& K^{(n)}=\frac{l}{6} \sum_{p=1}^{n-1}\left[-\frac{3}{4} K^{(p)} K^{(n-p)}+\Sigma_{\beta}^{(p) \alpha} \Sigma_{\alpha}^{(n-p) \beta}\right. \\
& \left.+\left[\begin{array}{c}
(4) \\
R
\end{array}\right]^{(n)}\right] \\
& K_{, y}^{(n)}-\frac{2}{l} K^{(n)}=\sum_{p=1}^{n-1}\left\{\frac{1}{4} K^{(p)} K^{(n-p)}+\Sigma^{(p) \alpha \beta} \Sigma_{\alpha \beta}^{(n-p)}\right\}, \\
& \sum_{\mu}^{(n) \lambda} \lambda_{\mid \lambda}-\frac{3}{4} K_{\mid \mu}^{(n)}+\sum_{p=1}^{n-1}\left\{\Gamma_{\lambda \alpha}^{(p) \alpha} \Sigma_{\mu}^{(n-p) \lambda}\right. \\
& \left.-\Gamma_{\alpha \mu}^{(p) \lambda} \Sigma^{(n-p)}{ }_{\lambda}^{\alpha}\right\}=0 .
\end{aligned}
$$

As one can see from Eq. (53), homogeneous solutions will appear at each order. However, we note that the subtlety discussed in the second order calculations never occurs in the higher order calculations. The existence of the infinite series is a manifestation of the nonlocality of the brane model. Therefore, we have two kinds of nonlocality on the brane. One is the nonlocality associated with homogeneous solutions and the other is the infinite series which is the reflection of the extent in the $y$ direction.

\section{EFFECTIVE EQUATIONS AND EFFECTIVE ACTION}

Now consider the consequences of the junction condition (10). The findings in this section are the following. We find the generalized dark radiation $\chi^{\mu}{ }_{\nu}$. The quadratic correction $\pi^{\mu}{ }_{\nu}$ is identified with $P^{\mu}{ }_{\nu}$ which is the local tensor defined later. The relation between the geometrical approach and the AdS/CFT approach is revealed. The brane effective action is obtained and the corresponding bulk geometry is given explicitly.

\section{A. Zeroth order}

From the zeroth order solution, we obtain

$$
\left.\left[K_{\nu}^{(0) \mu}-\delta_{\nu}^{\mu} K^{(0)}\right]\right|_{y=0}=-\frac{3}{l} \delta_{\nu}^{\mu}=-\frac{\kappa^{2}}{2} \sigma \delta_{\nu}^{\mu}
$$

Then we get the well known relation $\kappa^{2} \sigma=6 / l$. Here, we will assume that this relation holds exactly. It is apparent that $C^{\mu}{ }_{\nu}$ is not allowed to exist.

\section{B. First order}

Let us focus on the role of $\chi^{\mu}{ }_{\nu}$ in this part. At this order, the junction condition can be written as

$$
\left.\left[K_{\nu}^{(1) \mu}-\delta_{\nu}^{\mu} K^{(1)}\right]\right|_{y=0}=\frac{l}{2}\left(R_{\nu}^{\mu}-\frac{1}{2} \delta_{\nu}^{\mu} R\right)+\chi_{\nu}^{\mu}=\frac{\kappa^{2}}{2} T_{\nu}^{\mu} .
$$

Using the solutions obtained in the previous section and the formula

$E_{\nu}^{\mu}=K_{\nu, y}^{\mu}-\delta_{\nu}^{\mu} K_{, y}-K_{\lambda}^{\mu}{ }_{\lambda}^{\lambda}{ }_{\nu}+\delta_{\nu}^{\mu} K_{\beta}^{\alpha} K_{\alpha}^{\beta}-\frac{3}{l^{2}} \delta_{\nu}^{\mu}$,

we calculate the projective Weyl tensor as $E^{(1) \mu}{ }_{\nu}=2 / l \chi^{\mu}{ }_{\nu}$. Then we obtain the effective Einstein equation

$$
R_{\nu}^{\mu}-\frac{1}{2} \delta_{\nu}^{\mu} R=\frac{\kappa^{2}}{l} T_{\nu}^{\mu}-E_{\nu}^{(1) \mu} .
$$

At this order, we do not have the conventional Einstein equations. Recall that the dark radiation exists even in the low energy regime. Indeed, the low energy effective Friedmann equation becomes

$$
H^{2}=\frac{8 \pi G_{N}}{3} \rho+\frac{C}{a_{0}(t)^{4}},
$$

where $a_{0}(t), H$, and $C$ denote the scale factor on the brane, 
the Hubble constant, and a constant, respectively. This equation can be obtained from Eq. (60) by imposing the maximal symmetry on the spatial part of the brane world. Hence, we observe that $\chi^{\mu}{ }_{\nu}$ is the generalization of the dark radiation found in the cosmological context.

The conventional Einstein gravity can be recovered on the brane at this order when we adopt the boundary condition for which $\chi^{\mu}{ }_{\nu}$ vanish.

\section{Second order}

In this subsection, we assume $\chi_{\nu}^{\mu}=0$. Up to second order, the junction condition gives

$$
R_{\nu}^{\mu}-\frac{1}{2} \delta_{\nu}^{\mu} R+2 l^{2}\left[\tau_{\nu}^{\mu}+\left(\alpha-\frac{1}{4}\right) \mathcal{S}^{\mu}{ }_{\nu}+\frac{\beta}{3} \mathcal{K}^{\mu}{ }_{\nu}\right]=\frac{\kappa^{2}}{l} T^{\mu}{ }_{\nu} .
$$

Let us try to arrange the terms so as to reveal the geometrical meaning of the above equation. We can calculate the projective Weyl tensor as

$$
E_{\nu}^{(2) \mu}=l^{2}\left[P_{\nu}^{\mu}+2 \tau_{\nu}^{\mu}+2\left(\alpha-\frac{1}{4}\right) \mathcal{S}_{\nu}^{\mu}+\frac{2}{3} \beta \mathcal{K}^{\mu}{ }_{\nu}\right] .
$$

Substituting this expression into Eq. (62) yields our main result:

$$
G_{\mu \nu}^{(4)}=\frac{\kappa^{2}}{l} T_{\mu \nu}+l^{2} P_{\mu \nu}-E_{\mu \nu}^{(2)},
$$

where

$$
P_{\nu}^{\mu}=-\frac{1}{4} R_{\lambda}^{\mu} R_{\nu}^{\lambda}+\frac{1}{6} R R_{\nu}^{\mu}+\frac{1}{8} \delta_{\nu}^{\mu} R_{\beta}^{\alpha} R_{\alpha}^{\beta}-\frac{1}{16} \delta_{\nu}^{\mu} R^{2} .
$$

Notice that $E^{\mu}{ }_{\nu}$ contains the nonlocal part and the free parameters $\alpha$ and $\beta$. On the other hand, $P_{\nu}^{\mu}$ is determined locally. If we define $T_{\mu \nu}^{\mathrm{CFT}}=-2 l^{3} / \kappa^{2} \tau_{\mu \nu}$, we can write

$$
G_{\mu \nu}^{(4)}=8 \pi G_{N}\left(T_{\mu \nu}+T_{\mu \nu}^{\mathrm{CFT}}\right)-2 l^{2}\left(\alpha-\frac{1}{4}\right) \mathcal{S}_{\mu \nu}-\frac{2 l^{2}}{3} \beta \mathcal{K}_{\mu \nu} .
$$

It is possible to use the result of CFT at this point. For example, we can choose the $\mathcal{N}=4$ super Yang-Mills theory as the conformal matter. In that case, we simply put $\beta=0$. This is the way the AdS/CFT correspondence comes into the brane world scenario. Thus we get an explicit relation between the geometrical approach and the AdS/CFT approach. One can see the relationship in a different way. Within the accuracy we are considering, we can get $P^{\mu}{ }_{\nu}=\pi^{\mu}{ }_{\nu}$ using the lowest order equation $R_{\nu}^{\mu}=\kappa^{2} / l\left(T_{\nu}^{\mu}-1 / 2 \delta_{\nu}^{\mu} T\right)$. Hence, we can rewrite Eq. (64) as

$$
G_{\mu \nu}^{(4)}=8 \pi G_{N} T_{\mu \nu}+\kappa^{4} \pi_{\mu \nu}-E_{\mu \nu}^{(2)} .
$$

Now, the similarity between Eq. (2) and Eq. (67) is apparent. However, we note that our Eq. (67) is a closed system of equations provided that the specific conformal field theory is chosen.

Now we can read off the effective action as

$$
\begin{aligned}
S_{\mathrm{eff}}= & \frac{1}{16 \pi G_{N}} \int d^{4} x \sqrt{-h} R+S_{\text {matter }}+S_{\mathrm{CFT}} \\
& +\frac{\left(\alpha-\frac{1}{4}\right) l^{2}}{16 \pi G_{N}} \int d^{4} x \sqrt{-h}\left[R^{\mu \nu} R_{\mu \nu}-\frac{1}{3} R^{2}\right] \\
& +\frac{\beta l^{2}}{48 \pi G_{N}} \int d^{4} x \sqrt{-h} R^{2}
\end{aligned}
$$

where we have used the relations (45), (49), and (50) and we denoted the nonlocal effective action constructed from $\tau^{\mu}{ }_{\nu}$ as $S_{\text {CFT }}$. This effective action corresponds to the bulk geometry given by the metric

$$
\begin{aligned}
g_{\mu \nu}\left(y, x^{\mu}\right)= & a^{2}\left[h_{\mu \nu}-\frac{l^{2}}{2}\left(\frac{1}{a^{2}}-1\right)\left(R_{\mu \nu}-\frac{1}{6} h_{\mu \nu} R^{2}\right)+\frac{l^{3}}{4}\left(\frac{y}{a^{4}}-\frac{l}{4 a^{4}}+\frac{l}{a^{2}}-\frac{3 l}{4}\right) \mathcal{S}_{\mu \nu}-\frac{l^{4}}{2}\left(\frac{1}{a^{4}}-1\right)\right. \\
& \times\left(\tau_{\mu \nu}+\alpha \mathcal{S}_{\mu \nu}+\frac{\beta}{3} \mathcal{K}_{\mu \nu}\right)+\frac{l^{4}}{8}\left(\frac{1}{a^{4}}-1\right)\left(R_{\mu \lambda} R_{\nu}^{\lambda}-\frac{1}{2} R R_{\mu \nu}-\frac{1}{4} h_{\mu \nu} R_{\beta}^{\alpha} R_{\alpha}^{\beta}+\frac{5}{36} h_{\mu \nu} R^{2}\right)-\frac{l^{4}}{4}\left(\frac{1}{a^{2}}-1\right) \\
& \left.\times\left(R_{\mu \lambda} R_{\nu}^{\lambda}-\frac{1}{2} R R_{\mu \nu}-\frac{1}{12} h_{\mu \nu} R_{\beta}^{\alpha} R_{\alpha}^{\beta}+\frac{1}{12} h_{\mu \nu} R^{2}\right)\right] .
\end{aligned}
$$

This gives the holographic view of the bulk geometry. The bulk geometry can be reconstructed provided the additional knowledge of the nonlocal component $\tau^{\mu}{ }_{\nu}$ and the constants $\alpha$ and $\beta$ is available. Both represent the effects of the bulk geometry, which is apparent because they appear in the projective Weyl tensor $E^{\mu}{ }_{\nu}$. 


\section{CONCLUSION}

We developed a low energy iteration scheme for solving the equations of the brane world model. Using this formalism, we explicitly identified the low energy equations of motion describing gravity on the brane. The result should be useful in the investigation of various phenomena occurring in the brane world because it can treat nonlinear gravity as far as $l^{2} R(h) \ll 1$.

Our work was motivated by two important approaches, the geometric approach and the AdS/CFT approach. In fact, one of the purposes of this work was to clarify the relation between these approaches. In previous work, the trace anomaly of the CFT is identified with $\pi^{\mu}{ }_{\mu}$. This interpretation is rather paradoxical because, in the odd dimensional brane, no trace anomaly exists although $\pi_{\mu}^{\mu}$ exists (see the Appendix). We clarified this point by calculating the Weyl tensor. It turned out that, irrespective of dimensions, $\pi^{\mu}{ }_{\nu}$ corresponds to $P^{\mu}{ }_{\nu}$ at low energies. In the case of the fourdimensional brane, the trace part of $P^{\mu}{ }_{\nu}$ accidentally coincides with the trace anomaly of the CFT.

We found two kinds of nonlocality observed on the brane. One of them is encoded in the homogeneous solutions, and the other is found as an infinite series of the gradient expansion of our scheme. Thus even when we truncate the series at the second order, the knowledge of the homogeneous solutions is needed to solve the problem. Indeed, there are two homogeneous solutions because the system is described by a second order differential equation. One is used to satisfy the Dirichlet boundary condition at the brane. The other appears as the "dark" effects on the brane at each order of expansion in our scheme. At zeroth order, $C^{\mu}{ }_{\nu}$ appears. However, this term must vanish, from consistency. It is at first order that the generalized dark radiation term $\chi^{\mu}{ }_{\nu}$ appeared. This term reduces to the dark radiation in the effective Friedmann equation under the assumption of homogeneity. Note that it is possible to put $\chi^{\mu}{ }_{\nu}=0$ if one prefers. This can be achieved by putting the black hole mass to zero in the cosmological case. As for the general cases, further consideration is needed. At second order, we deduced the nonlocal component $\tau^{\mu}{ }_{\nu}$ from the homogeneous solution. At this time, it is far from possible to put $\tau^{\mu}{ }_{\nu}=0$ without losing consistency. We must treat it as an integro-differential equation [35] or coupled equations.

Needless to say, the ambiguity of the effective action comes from the variety of the bulk geometry. We have given the explicit correspondence between the effective action and the bulk metric, which could give a holographic view of the brane world. Of course, this ambiguity should be fixed by proper consideration of the boundary condition in the bulk [36]. Once the boundary condition is determined, we can attack various astrophysical and cosmological problems.

As an application of our results, it would be interesting to consider the nature of the gravitational wave in the brane world. It is also important to investigate the quantum brane world from this point of view. In particular, we will apply our formalism to the inflation model driven by a bulk scalar field $[37,38]$. The analysis of the present paper can be ex- tended to the two brane system. In particular, the low energy dynamics of the radion could be treated by means of our method [39]. We will also study more general models like the Horava-Witten model in the future.

\section{ACKNOWLEDGMENTS}

We would like to thank the participants of the YITP workshop YITP-W-01-15 on "Braneworld-Dynamics of Spacetime Boundary" for fruitful discussions. This work was supported in part by Monbukagakusho Grant-in-Aid No. 14540258 .

\section{APPENDIX: $(d+1)$-DIMENSIONAL RESULTS $(d \neq 4)$}

The qualitative consequences of the low energy expansion scheme depend on the dimensions. Hence, for completeness, we investigated the $(d+1)$-dimensional problem.

We obtain basic equations which hold in the bulk as follows:

$$
\begin{aligned}
\Sigma_{\nu, y}^{\mu}-K \Sigma_{\nu}^{\mu} & =-\left[\begin{array}{c}
(d) \\
R^{\mu}{ }_{\nu}-\frac{1}{d} \delta_{\nu}^{\mu} R
\end{array}\right], \\
\frac{d-1}{d} K^{2}-\Sigma^{\alpha}{ }_{\beta} \Sigma^{\beta}{ }_{\alpha} & =R^{(d)}+\frac{d(d-1)}{l^{2}}, \\
K_{, y}-\frac{1}{d} K^{2}-\Sigma^{\alpha \beta} \Sigma_{\alpha \beta} & =-\frac{d}{l^{2}}, \\
\nabla_{\lambda} \Sigma_{\mu}{ }^{\lambda}-\frac{d-1}{d} \nabla_{\mu} K & =0 .
\end{aligned}
$$

Since the calculations are similar to those in the $d=4$ case, we simply write down the results in the following subsections.

\section{Zeroth order}

At the zeroth order, we have

$$
K^{(0)}=\frac{d}{l} \text {. }
$$

The zeroth order metric is given by

$$
d s^{2}=d y^{2}+a^{2}(y) h_{\mu \nu}\left(x^{\mu}\right) d x^{\mu} d x^{\nu}, \quad a(y)=e^{-2 y / l} .
$$

\section{First order}

At first order, the solutions are

$$
K^{(1)}=\frac{l}{2(d-1) a^{2}} R(h),
$$




$$
\Sigma_{\nu}^{(1) \mu}=\frac{l}{(d-2) a^{2}}\left(R_{\nu}^{\mu}-\frac{1}{d} \delta_{\nu}^{\mu} R\right)+\frac{\chi_{\nu}^{\mu}}{a^{d}}
$$

where $\chi_{\nu}^{\mu}$ is a homogeneous solution which satisfies $\chi_{\mu}^{\mu}$ $=0$ and $\chi^{\mu}{ }_{\nu \mid \mu}=0$. This corresponds to the dark radiation at this order. The metric can be deduced as

$$
\begin{aligned}
g_{\mu \nu}^{(1)}= & -\frac{l^{2}}{d-2}\left(\frac{1}{a^{2}}-1\right)\left(R_{\mu \nu}-\frac{1}{2(d-1)} h_{\mu \nu} R\right) \\
& -\frac{2 l}{d}\left(\frac{1}{a^{d}}-1\right) \chi_{\mu \nu},
\end{aligned}
$$

where we have imposed the boundary condition $g_{\mu \nu}^{(1)}(y$ $\left.=0, x^{\mu}\right)=0$.

\section{Second order}

If we ignore the $\chi$ field then we get the following results:

$$
\begin{aligned}
& K^{(2)}=\frac{l^{3}}{2(d-2)^{2} a^{4}}\left(R_{\beta}^{\alpha} R_{\alpha}^{\beta}-\frac{3 d-4}{4(d-1)^{2}} R^{2}\right)-\frac{l^{3}}{2(d-1)(d-2) a^{2}}\left(R_{\beta}^{\alpha} R_{\alpha}^{\beta}-\frac{1}{2(d-1)} R^{2}\right) . \\
& \Sigma_{\nu}^{(2) \mu}=\frac{l^{3}}{(d-2)(d-4) a^{4}}\left[R^{\mu}{ }_{\alpha} R^{\alpha}{ }_{\nu}-\frac{1}{d-1} R^{\mu}{ }_{\nu}-\frac{1}{2}\left(R_{\mid \nu \alpha}^{\alpha \mu}+\left.R_{\nu}^{\alpha \mid \mu}\right|_{\alpha}\right)+\frac{1}{2} \square R^{\mu}{ }_{\nu}+\frac{d}{4(d-1)} R^{\mid \mu}{ }_{\mid \nu}-\frac{1}{4(d-1)} \delta_{\nu}^{\mu} \square R\right. \\
& \left.-\frac{1}{d} \delta_{\nu}^{\mu} R_{\beta}^{\alpha} R_{\alpha}^{\beta}+\frac{1}{d(d-1)} \delta_{\nu}^{\mu} R^{2}\right]+\frac{l^{3}}{a^{d}} t_{\nu}^{\mu}-\frac{l^{3}}{(d-2)^{2} a^{2}}\left[R_{\alpha}^{\mu} R_{\nu}^{\alpha}-\frac{1}{2(d-1)} R R_{\nu}^{\mu}-\frac{1}{2}\left(R_{\mid \nu \alpha}^{\alpha \mu}+R_{\nu \mid \alpha}^{\alpha \mid \mu}\right)\right. \\
& \left.+\frac{1}{2} \square R_{\nu}^{\mu}+\frac{d}{4(d-1)} R_{\mid \nu}^{\mid \mu}-\frac{1}{4(d-1)} \delta_{\nu}^{\mu} \square R-\frac{1}{d} \delta_{\nu}^{\mu} R_{\beta}^{\alpha} R_{\alpha}^{\beta}+\frac{1}{2 d(d-1)} \delta_{\nu}^{\mu} R^{2}\right],
\end{aligned}
$$

where the homogeneous solution $t^{\mu}{ }_{\nu}$ satisfies the transverse and traceless conditions

$$
t_{\nu \mid \mu}^{\mu}=0, \quad t_{\mu}^{\mu}=0
$$

This is the point where the dependence on the dimensions appears. We do not have a trace anomaly, in contrast to the case of $d=4$.

\section{Effective equations and effective action}

The consequences of the junction condition (10), order by order, are the following. At zeroth order, we have

$$
\left.\left[K_{\nu}^{(0) \mu}-\delta_{\nu}^{\mu} K^{(0)}\right]\right|_{y=0}=-\frac{d-1}{l}=-\frac{\kappa^{2}}{2} \sigma \delta_{\nu}^{\mu} .
$$

Thus, we get the relation, $\kappa^{2} \sigma=2(d-1) / l$ and assume that this relation holds exactly.

At first order, we obtain

$$
\left.\left[K_{\nu}^{(1) \mu}-\delta_{\nu}^{\mu} K^{(1)}\right]\right|_{y=0}=\frac{l}{d-2}\left(R_{\nu}^{\mu}-\frac{1}{2} \delta_{\nu}^{\mu} R\right)+\chi_{\nu}^{\mu}=\frac{\kappa^{2}}{2} T_{\nu}^{\mu}
$$

The homogeneous solution $\chi_{\mu \nu}$ is the generalized dark radiation. Supposing the relation $8 \pi G_{N}=(d-2) \kappa^{2} / 2 l$ holds, then the conventional Einstein equation can be recovered on the brane at this order when we put $\chi_{\mu \nu}=0$. This can be performed without losing consistency.

Finally, up to second order, the junction condition gives 


$$
\begin{gathered}
\frac{l}{d-2}\left(R_{\nu}^{\mu}{ }_{\nu}-\frac{1}{2} \delta_{\nu}^{\mu} R\right)+\frac{l^{3}}{(d-2)^{2}(d-4)}\left[2 R_{\alpha}^{\mu} R_{\nu}^{\alpha}-\frac{d}{2(d-1)} R R_{\nu}^{\mu}-\left(R_{\mid \nu \alpha}^{\alpha \mu}+\left.R_{\nu}^{\alpha}\right|_{\alpha}\right)+\square R_{\nu}^{\mu}+\frac{d}{2(d-1)} R_{\mid \nu}^{\mid \mu}\right. \\
\left.-\frac{1}{2(d-1)} \delta_{\nu}^{\mu} \square R-\frac{1}{2} \delta_{\nu}^{\mu} R_{\beta}^{\alpha} R_{\alpha}^{\beta}+\frac{d}{8(d-1)} \delta_{\nu}^{\mu} R^{2}\right]=\frac{\kappa^{2}}{2} T_{\nu}^{\mu}{ }_{\nu}
\end{gathered}
$$

We calculate the projective Weyl tensor to find the geometrical meaning of the above equation as

$$
\begin{aligned}
E_{\nu}^{(2) \mu}= & \frac{d l^{2}}{(d-2)^{2}(d-4)}\left[R_{\alpha}^{\mu} R_{\nu}^{\alpha}-\frac{1}{d-1} R_{\nu}^{\mu}{ }_{\nu}-\frac{d-2}{d}\left(R_{\mid \nu \alpha}^{\alpha \mu}+R_{\nu \mid \alpha}^{\alpha \mid \mu}\right)+\frac{d-2}{d} \square R_{\nu}^{\mu}+\frac{d-2}{2(d-1)} R_{\mid \nu}^{\mid \mu}\right. \\
& \left.-\frac{d-2}{2 d(d-1)} \delta_{\nu}^{\mu} \square R-\frac{1}{d} \delta_{\nu}^{\mu} R_{\beta}^{\alpha} R_{\alpha}^{\beta}+\frac{1}{d(d-1)} \delta_{\nu}^{\mu} R^{2}\right] .
\end{aligned}
$$

Substituting this expression into Eq. (A15) yields our main result

$$
G_{\mu \nu}^{(4)}=\frac{(d-2) \kappa^{2}}{2 l} T_{\mu \nu}+l^{2} P_{\mu \nu}-E_{\mu \nu}^{(2)},
$$

where

$$
P_{\nu}^{\mu}=-\frac{1}{(d-2)^{2}}\left[R_{\lambda}^{\mu} R_{\nu}^{\lambda}-\frac{d}{2(d-1)} R R_{\nu}^{\mu}-\frac{1}{2} \delta_{\nu}^{\mu} R^{\alpha}{ }_{\beta} R_{\alpha}^{\beta}+\frac{d+2}{8(d-1)} \delta^{\mu}{ }_{\nu} R^{2}\right] .
$$

Within the accuracy we are considering, using the lowest order equation $R^{\mu}{ }_{\nu}=\kappa^{2} / l\left[(d-2) / 2 T^{\mu}{ }_{\nu}-1 / 2 \delta_{\nu}^{\mu} T\right]$, we get formally the same result as that of Shiromizu et al.:

$$
G_{\mu \nu}^{(4)}=8 \pi G_{N} T_{\mu \nu}+\kappa^{4} \pi_{\mu \nu}-E_{\mu \nu}^{(2)},
$$

with

$$
\pi_{\mu \nu}=-\frac{1}{4} T_{\mu}^{\lambda} T_{\lambda \nu}+\frac{1}{4(d-1)} T T_{\mu \nu}+\frac{1}{8} g_{\mu \nu}\left(T^{\alpha \beta} T_{\alpha \beta}-\frac{1}{d-1} T^{2}\right) .
$$

Thus, we have established the correspondence between the geometrical and AdS/CFT approaches in any dimensions.

[1] L. Randall and R. Sundrum, Phys. Rev. Lett. 83, 4690 (1999).

[2] J. Garriga and T. Tanaka, Phys. Rev. Lett. 84, 2778 (2000).

[3] S. Giddings, E. Katz, and L. Randall, J. High Energy Phys. 03, 023 (2000).

[4] H.A. Chamblin and H.S. Reall, Nucl. Phys. B562, 133 (1999).

[5] N. Kaloper, Phys. Rev. D 60, 123506 (1999).

[6] K. Koyama and J. Soda, Phys. Lett. B 483, 432 (2000).

[7] P. Binétruy, C. Deffayet, U. Ellwanger, and D. Langlois, Phys. Lett. B 477, 285 (2000).

[8] E. Flanagan, S. Tye, and I. Wasserman, Phys. Rev. D 62, 024011 (2000).

[9] P. Kraus, J. High Energy Phys. 12, 011 (1999).

[10] S. Mukohyama, Phys. Lett. B 473, 241 (2000).

[11] D. Ida, J. High Energy Phys. 09, 014 (2000).

[12] K. Koyama and J. Soda, Phys. Rev. D 62, 123502 (2000).

[13] S. Mukohyama, Phys. Rev. D 62, 084015 (2000).

[14] H. Kodama, A. Ishibashi, and O. Seto, Phys. Rev. D 62, 064022 (2000).
[15] C. van de Bruck, M. Dorca, R.H. Brandenberger, and A. Lukas, Phys. Rev. D 62, 123515 (2000).

[16] D. Langlois, Phys. Rev. D 62, 126012 (2000).

[17] R. Maartens, Phys. Rev. D 62, 084023 (2000).

[18] N. Deruelle, T. Dolezel, and J. Katz, Phys. Rev. D 63, 083513 (2001); B. Leong, P. Dunsby, A. Challinor, and A. Lasenby, ibid. 65, 104012 (2002).

[19] K. Uzawa and J. Soda, Mod. Phys. Lett. A 16, 1089 (2001).

[20] K. Koyama and J. Soda, Phys. Rev. D 65, 023514 (2002).

[21] D. Langlois, R. Maartens, M. Sasaki, and D. Wands, Phys. Rev. D 63, 084009 (2001).

[22] T. Shiromizu, K. Maeda, and M. Sasaki, Phys. Rev. D 62, 024012 (2000).

[23] S.S. Gubser, Phys. Rev. D 63, 084017 (2001); L. Anchordoqui, C. Nunez, and K. Olsen, J. High Energy Phys. 10, 050 (2000).

[24] J. Maldacena, Adv. Theor. Math. Phys. 2, 231 (1998).

[25] S.W. Hawking, T. Hertog, and H.S. Reall, Phys. Rev. D 62 , 
043501 (2000); S. Nojiri, S.D. Odintsov, and Sergio Zerbini, ibid. 62, 064006 (2000); S. Nojiri and S.D. Odintsov, Phys. Lett. B 484, 119 (2000).

[26] M. Henningson and K. Skenderis, J. High Energy Phys. 07, 023 (1998); S. Nojiri and S.D. Odintsov, Phys. Lett. B 444, 92 (1998); S. Nojiri, S.D. Odintsov, and S. Ogushi, Phys. Rev. D 62, 124002 (2000).

[27] S. de Haro, K. Skenderis, and S.N. Solodukhin, Commun. Math. Phys. 217, 595 (2001).

[28] S. de Haro, K. Skenderis, and S.N. Solodukhin, Class. Quantum Grav. 18, 3171 (2001).

[29] K. Koyama and J. Soda, J. High Energy Phys. 05, 027 (2001); N.S. Deger and A. Kaya, ibid. 05, 030 (2001); J.P. Gregory and A. Padilla, hep-th/0204218.

[30] T. Shiromizu and D. Ida, Phys. Rev. D 64, 044015 (2001).
[31] K. Tomita, Prog. Theor. Phys. 54, 730 (1975).

[32] G.L. Comer, N. Deruelle, D. Langlois, and J. Parry, Phys. Rev. D 49, 2759 (1994).

[33] D.S. Salopek and J.M. Stewart, Phys. Rev. D 47, 3235 (1993).

[34] J. Soda, H. Ishihara, and O. Iguchi, Prog. Theor. Phys. 94, 781 (1995).

[35] S. Mukohyama, Phys. Rev. D 64, 064006 (2001); 65, 084036 (2002).

[36] T. Wiseman, Phys. Rev. D 65, 124007 (2002).

[37] S. Kobayashi, K. Koyama, and J. Soda, Phys. Lett. B 501, 157 (2001).

[38] Y. Himemoto and M. Sasaki, Phys. Rev. D 63, 044015 (2001).

[39] S. Kanno and J. Soda, Phys. Rev. D (to be published), hep-th/0207029; T. Wiseman, Class. Quantum Grav. 19, 3083 (2002). 